\title{
Villes et villages de Haute-Bretagne (XIe-début XIVe siècles)
}

\section{Magali Watteaux}

\section{(2) OpenEdition}

\section{Journals}

Édition électronique

URL : http://journals.openedition.org/rao/3192

DOI : 10.4000/rao.3192

ISBN : 978-2-7535-5014-8

ISSN : $1775-3732$

\section{Éditeur}

Presses universitaires de Rennes

\section{Édition imprimée}

Date de publication : 31 décembre 2015

ISBN : 978-2-7535-5012-4

ISSN : 0767-709X

Référence électronique

Magali Watteaux, "Villes et villages de Haute-Bretagne (Xle-début XIVe siècles) 》, Revue archéologique de l'Ouest [En ligne], 32 | 2015, mis en ligne le 28 avril 2016, consulté le 02 mars 2021. URL : http:// journals.openedition.org/rao/3192 ; DOI : https://doi.org/10.4000/rao.3192 
de restituer un pilier votif dont la hauteur peut être estimée à environ $9 \mathrm{~m}$ et qui avait fait l'objet d'une première étude en 1972. Dédié à Jupiter et supporté par un piédestal, il est constitué de trois niveaux sculptés en bas-relief figurant en alternance des divinités et des scènes mythologiques. S'y ajoutent 3 blocs d'une dédicace à Minerve et aux divinités des empereurs. Cette inscription témoigne du financement de la construction d'un temple par deux frères, citoyens romains, suivant la pratique courante de l'évergésie. Les autres blocs n'avaient pas, jusqu'alors, fait l'objet d'une publication. 24 d'entre eux appartiennent à un temple de forme polygonale, dont l'architrave et une partie des pilastres et de l'entablement peuvent être restituées. 2 autres, enfin, participent du couronnement d'un autel réservé aux sacrifices lors des cérémonies.

L'ensemble fait l'objet d'une analyse minutieuse qui combine texte, dessins et photographies, parmi lesquelles plusieurs clichés inédits issus des archives du père de la Croix. L'enquête est complétée par une approche des techniques de taille et de mise en ouvre de ces blocs en calcaire et se conclut par une comparaison pertinente avec le sanctuaire picton du Gué-de-Sciaux à Antigny (Vienne). Celui d'Yzeures-sur-Creuse, dont les restes trahissent l'ampleur et la qualité des ornamenta, se trouvait quant à lui en limite sud du territoire turon, au sein d'une probable agglomération antique. Les datations stylistiques suggèrent une édification du temple et du pilier votif à la fin du $\mathrm{II}^{\mathrm{e}}$ siècle ou au début du III ${ }^{\mathrm{e}}$ siècle apr. J.-C.

Conçu tout à la fois comme une étude archéologique complète et rigoureuse mais aussi comme un guide proposé aux visiteurs du musée, cet ouvrage au prix modique s'adresse à tous ceux qui s'intéressent à l'architecture religieuse des Gaules romaines, mais aussi à l'histoire de l'archéologie, illustrée ici par la reproduction de nombreuses photographies prises au moment des fouilles de 1896 et par la narration des conditions dans lesquelles celles-ci se déroulèrent.

Martial MonTeIL

(université de Nantes - LARA-UMR 6566 CReAAH)

BaCHELIER Julien, 2014 - Villes et villages de Haute-Bretagne (XI'-début XIV siècles). Analyses morphologiques, supplément AK des Dossiers du Centre Régional d'Archéologie d'Alet, 242 p. (ISSN 0399-6662).

Cette publication est issue des annexes d'une thèse d'histoire médiévale ${ }^{1}$, soutenue en 2013 à Rennes 2 sous la direction de Daniel Pichot, qui en signe la préface. L'auteur, agrégé d'histoire, y présente l'ensemble des plans de villes, bourgs et villages - voire fermes - sur lesquels il a travaillé, en Haute-Bretagne.

Eu égard au nombre de plans analysés et présentés, cet ouvrage est avant tout un atlas qui constitue la base des analyses morphologiques proposées. La partie cartographique représente, pour cette raison, la majeure partie du livre (195 pages sur 242). La précède une "présentation » d'ordre méthodologique et conclusive (mais assez courte : 17 pages) abordant : le travail de constitution des données (p. 11-13), une brève historiographie des recherches en morphologie (p. 14-18), le vocabulaire utilisé dans les fiches établies par localité (p. 19), les difficultés de l'exercice (p. 19-20) et les principaux enseignements que l'auteur tire de ce travail (p. 21-24). Puis suivent les fiches des villes, bourgs et villages de Haute-Bretagne connus dans les sources écrites entre le $\mathrm{XI}^{\mathrm{e}}$ et le début du XIV $^{e}$ siècle. On découvre 173 entrées, par nom de lieux, associant une notice et un plan. Chaque notice adopte

1. «Villes et villages de Haute-Bretagne. Les réseaux de peuplement $\left(\mathrm{XI}^{\mathrm{e}}-\mathrm{XIII}^{\mathrm{e}} \mathrm{s}.\right) »$. une présentation normalisée la situation administrative (département, arrondissement, canton) et ecclésiastique durant les $\mathrm{XI}^{\mathrm{e}}$-XIII ${ }^{\mathrm{e}}$ siècles (diocèse, doyenné), la superficie du site étudié ${ }^{2}$, les analyses morphologiques réalisées par l'auteur et les sources et références bibliographiques à partir desquelles il a travaillé. À ces notices est associé un plan, dessiné sous Adobe Illustrator ${ }^{3}$, à partir du levé du cadastre napoléonien qui représente " la plus ancienne représentation cartographique englobant de manière précise et détaillée toutes les localités étudiées " (p. 13). Si très ponctuellement d'autres documents cartographiques ont été exploités ${ }^{4}$, le cadastre ancien reste la base des analyses morphologiques. L'ouvrage se clôt sur une liste des abréviations, des sources et de la bibliographie.

L'objectif affiché de J. Bachelier est de pallier la sousexploitation du cadastre napoléonien en histoire médiévale qui demeure " peu utilisé » hors des études monographiques et " encore trop souvent dans un seul but illustratif» (p. 9).

2. Mais la méthode utilisée n'est pas précisée alors que l'exercice est délicat pour les agglomérations à la structure éclatée et à l'extension diffuse.

3. On regrettera à ce sujet que ce travail, pourtant commencé au début des années 2000, n'ait pas été réalisé sous SIG afin d'en faire un outil susceptible d'être partagé et enrichi plus facilement.

4. Une copie couleurs sur feuille libre d'un plan de Fougères du $\mathrm{XVIII}^{\mathrm{e}}$ siècle (date non communiquée) est d'ailleurs jointe à la publication. 
L'auteur est par ailleurs motivé par le manque de sources écrites et archéologiques en Haute-Bretagne alors que la période envisagée semble être " au cour des principales dynamiques de peuplement " (ibid.). Il souhaite enfin mettre l'accent sur les petites villes, bourgs et villages qui sont, sinon les oubliés de l'histoire et de l'archéologie urbaines, $\mathrm{du}$ moins les parents pauvres de ces recherches. C'est particulièrement vrai en Haute-Bretagne où J. Bachelier souligne la "méconnaissance de la topographie de ces villes et villages " (p. 11). Néanmoins il n'entend pas en rester à une approche de topographie historique classique et souhaite proposer une analyse de la morphogenèse : «nous nous sommes attaché à décrire les formes pour tenter de comprendre comment sont nées et se sont développées les agglomérations » (p. 10). Pour cela il reconnaît s'inspirer beaucoup du travail de Bernard Gauthiez et de ses collègues sur les bourgs ruraux en Touraine.

Avant de rentrer dans le détail de la critique, on notera pour le regretter que la publication en tant qu'objet ne soit pas de la plus belle des factures. Outre une mise en page un brin austère - mais que nous ne critiquerons pas eu égard au caractère local de la maison d'édition qui accueille généreusement cette publication au format inhabituel - on s'étonnera surtout des coquilles (dont une en couverture intérieure) et même de copier-coller mal digérés sur la $4^{\mathrm{e}}$ de couverture. Une relecture plus attentive aurait par ailleurs permis de soigner la rédaction qui montre un usage souvent erroné de la ponctuation (virgules en particulier). S'agissant des plans, la résolution de certains laisse parfois à désirer comme le plan $\mathrm{n}^{\circ} 32$, limitant de ce fait les observations que le lecteur peut faire par lui-même. Plus fâcheux, quelques manques et erreurs se glissent parfois. Ainsi sur le plan $n^{\circ} 1$ on ne voit pas les numéros cités dans l'analyse et, inversement, sur le plan $\mathrm{n}^{\circ} 2$ sont portés des numéros qui ne sont pas repris dans l'analyse. L'articulation entre l'image et le texte est donc laissée à l'appréciation du lecteur. Autres exemples de ces problèmes malheureux de forme : le plan d'Acigné-bourg (plan $n^{\circ} 2$ ) a été inversé avec d'AcignéBourgon (plan $\mathrm{n}^{\circ} 1$ ); le nord est mal indiqué sur le plan $\mathrm{n}^{\circ} 3$ qui est en fait orienté à l'est; le plan n ${ }^{\circ} 33$ (Châteaugironville) est imprimé deux fois, à la place du plan $n^{\circ} 34$ qui devrait être celui de Veneffles (il manque donc le plan de Veneffles); idem pour les plans $n^{\circ} 126$ de Ruffiac et $n^{\circ} 127$ de Sains (il manque donc le plan de Sains).

Sur le fond, ma critique portera plus sur la méthodologie de l'analyse morphologique que sur les conclusions qu'en tire l'auteur sur les dynamiques de peuplement car ces dernières ne sont exposées que très brièvement et font partie du corps de la thèse, qui sera publié plus tard. Mais avant cela, disons un mot des sites retenus pour l'analyse. Le corpus étudié par J. Bachelier est très hétérogène - de la cité rennaise à l'abbaye fondée au milieu des bois - parce qu'il recouvre toutes les localités considérées comme « lieux centraux » mentionnées dans les textes (près de 3000 actes et quelques œuvres littéraires des XI ${ }^{\mathrm{e}}$-XIII ${ }^{\mathrm{e}}$ siècles). Pour définir ces " centres de pouvoir et pas seulement au plan politique ", l'auteur emprunte sa méthode à la thèse de Jean-Luc Fray sur la Lorraine médiévale et élabore une grille d'analyse très intéressante hiérarchisant l'importance de ces lieux religieux, castraux, économiques et agglomérés (p. 11). L'auteur a recentré sa liste « sur les localités disposant d'au moins deux critères de centralité, bien souvent il s'agissait de l'église paroissiale et d'un prieuré, mais d'autres combinaisons se dessinent associant le lieu de culte communautaire à un marché, une foire, un château, majeur ou secondaire, un burgus» (ibid.). Si la démarche initiale est compréhensible, on s'étonne cependant de trouver dans les notices certains sites qui ne semblent pas être de tels « lieux centraux ». Ainsi, Rallion ou Allion (commune de La Bouëxière) correspond simplement à un cimetière mérovingien puis à un prieuré ou une collégiale au XIII ${ }^{\mathrm{e}}$ siècle et, enfin, à une chapelle et quelques bâtiments des $\mathrm{XV}^{\mathrm{e}}$-XVI ${ }^{\mathrm{e}}$ siècles (p. 45). Doit-on finalement comprendre que les critères de centralité doivent être a minima deux et ce quelle que soit leur chronologie? Le plan ne montre que quatre bâtiments et l'auteur souligne l'isolement du site qui apparaît donc bien peu central! Même cas de figure au Breteil (Le Ménéhil) où l'on ne trouve qu'une ferme isolée (p. 52). Enfin, l'utilisation du concept des " lieux centraux " devrait amener à réfléchir $\mathrm{au}(\mathrm{x})$ territoire(s) qu'ils dessinent. Cette analyse du corpus à une autre échelle - en tant que points dans l'espace occupant une place les uns par rapports aux autres - n'est pas abordée ici; probablement doit-elle faire l'objet d'un développement spécifique dans la publication spécifique du corps de la thèse. Ce sera donc sur les analyses purement morphologiques que je m'attarderai.

On note tout d'abord, à l'instar du corpus, une certaine hétérogénéité dans les analyses. Si l'on comprend facilement que certains villages offrent une matière insuffisante, on comprend moins la quasi absence d'analyse morphologique pour la ville de Dinan ou celle encore de Saint-Malo (plan $\mathrm{n}^{\circ} 145$ ) où l'on décèle, pourtant, au premier coup d'œil de beaux lotissements dans les anciens remparts, qui auraient justifié une analyse approfondie au regard de la densité urbaine. Au contraire, la ville de Rennes tranche par le nombre de pages qui lui est consacrée (et l'on comprend aisément pourquoi) alors que, pourtant, l'analyse ne dépasse pas le stade d'observations générales de topographie historique. On s'étonne également que certains plans ne

5. Je ne parle pas de topographie historique, qui semble prendre le relais dans ce cas. 
comportent aucune proposition d'interprétation alors qu'il n'y a pas moins de choses à observer que sur d'autres plans pourtant commentés, comme par exemple sur le plan n ${ }^{\circ} 26$ où on lit parfaitement l'effet polarisateur opéré par l'église sur les chemins et sur l'habitat qui se groupe majoritairement autour. En outre, il manque souvent des arguments pour étayer telle ou telle information. Ainsi cette " ancienne voie " mentionnée sans autre précision sur le plan $n^{\circ} 2$ (Acigné) ou - plus problématique - le cas des voies antiques comme celle évoquée à Bain-de-Bretagne. La bibliographie mobilisée pour avancer cette hypothèse (Guillotin de Corson et P. Banéat) devrait pourtant inviter à la prudence car toutes leurs indications sur les voies romaines sont à prendre avec les plus grandes précautions et donc à réévaluer au cas par cas. On pourrait faire la même critique à propos de telle motte datée " très probablement des siècles centraux du Moyen Âge " (p. 164) ou de telle autre "située près d'un lieu de culte " mais qu'on ne voit pas sur le plan (ibid.). Une meilleure articulation entre certaines informations historiques et la bibliographie permettrait donc de donner plus de poids aux hypothèses avancées. Ce constat va de pair avec un certain flou, parfois, dans les interprétations proposées par l'auteur. Ainsi, le centre d'Acigné est-il qualifié par J. Bachelier de " faiblement polarisateur et structuré " (p. 30) alors qu'on observe pas moins de huit chemins qui convergent vers le centre et son église et que ce dernier présente une structure très nette - et très classique - de village-rue. On en vient donc à s'interroger sur le sens exact, sous la plume de l'auteur, des concepts de polarisation et structuration.

De manière plus générale, les analyses pêchent par une approche monoscalaire et la sous-exploitation de l'information viaire, les deux problèmes étant liés. La cartographie centrée sur les agglomérations amène à occulter certaines réalités pourtant essentielles à l'analyse que sont les logiques de desserte et de connexions entre sites. Changer d'échelle permet ainsi d'intégrer certaines données archéologiques importantes comme les voies romaines qui se situent à proximité de tel ou tel site. Le cas de Châteaugiron est éclairant : la présence de la voie romaine Rennes-Angers - bien attestée archéologiquement et morphologiquement -, juste au sud du bourg castral, semble déterminante dans le choix du site qu'occupe ce bourg, qui capte à son profit le trafic de cette voie de grand parcours ${ }^{6}$. Il est donc dommage de ne pas figurer cette voie. Il aurait fallu également ajouter aux plans les noms et/ou aboutissants des chemins mentionnés sur le cadastre. Ainsi, avec l'indication sur le plan $n^{\circ} 3 \mathrm{du}$ « chemin vicinal de Châteaugiron à Amanlis "

6. Voie qui, avec la voie Rennes-Le Mans, est également indispensable à l'étude du site d'habitat altomédiéval de la Perdriotais - évoqué par l'auteur p. 62 , juste au sud du bourg. on verrait mieux en contrepoint se dessiner un ancien chemin, qui passait devant l'église, ce qui constitue donc un élément de datation relative. Ailleurs, à Saint-Aubindu-Pavail (plan n ${ }^{\circ} 130$ ) par exemple, la structure " éclatée " du bourg, relevée par l'auteur, pourrait en partie trouver son explication dans le réseau routier : en effet au nord du chemin est-ouest menant à l'église se trouve, parallèle, le grand chemin de Châteaugiron à La Guerche-de-Bretagne, qui supporte un trafic bien plus important et qui a donc probablement joué à un rôle d'attracteur expliquant le groupe de maisons décalées vers le nord par rapport au centre ecclésial.

L'absence de certaines références bibliographiques est, en outre, surprenante. Ainsi le manuel des sources et techniques de l'archéogéographie, dirigé par Sandrine Robert $^{7}$, aurait aidé J. Bachelier dans ses analyses, de même que les publications récentes de Gérard Chouquer sur la morphologie urbaine en particulier et sur l'analyse des formes en général (depuis 2003 des dizaines de publications sont parues), lui auraient permis d'améliorer son historiographie. On s'étonnera plus encore de la sous-représentation des travaux d'Hélène Noizet ${ }^{8}$, historienne médiéviste pourtant incontournable sur la morphologie urbaine, et de la thèse de Cédric Lavigne9, l'un des rares médiévistes à avoir travaillé sur les planifications médiévales, certes en contexte rural mais selon une méthodologie morphologique et en apportant des résultats précieux pour qui souhaite étudier la morphologie du second Moyen Âge.

Ce changement d'échelle, s'il est très utile voire indispensable à l'analyse et l'interprétation morphologiques, représente un très gros investissement en temps, peu compatible avec le traitement d'un corpus de 173 agglomérations. Ce parti-pris initial s'explique par le point de départ méthodologique de la thèse : "partir des sources écrites" (p. 24) et les confronter, dans un second temps, avec les plans parcellaires du cadastre ancien. Si cette démarche n'est pas inintéressante, loin s'en faut, elle touche cependant rapidement ses limites car la planimétrie comporte de multiples héritages, qui renvoient à des réalités variées et des périodes différentes, compréhensibles uniquement via une analyse diachronique et multiscalaire. Cela demande donc de partir du plan et non du texte puis, dans un second temps, d'opérer des va-et-vient entre les différents types de documentation. Cela demande également de se plonger

7. Robert S., Sources et techniques de l'archéogéographie, Besançon, Presses universitaires de Franche-Comté, 2011.

8. Son dernier livre Paris de parcelles en pixels (Paris, PUV, 2013) est d'ailleurs mal référencé puisque c'est elle qui a dirigé la publication et non Boris Bove, qui est second éditeur.

9. Lavigne C., Essai sur la planification agraire au Moyen Âge. Les paysages neufs de la Gascogne médiévale (XIIT-XIV siècles), Bordeaux, Éditions Ausonius, coll. «Scripta varia; 5 », 2002. 
dans l'histoire locale et, pour cela, de lire beaucoup plus de travaux que les grandes synthèses vieillies des érudits-stars bretons. Ainsi, l'étude de la morphogenèse de Châteaugiron et de Veneffles - pour reprendre ces exemples que je connais mieux - gagnent à intégrer les informations sur les voies anciennes, comme nous l'avons dit, mais aussi le fait que le second, au sud du premier, est un centre paroissial très ancien, dont le territoire fut démantelé au profit de Châteaugiron ${ }^{10}$. On comprend mieux également le dessin du réseau viaire à Veneffles, qui traduit le déclin (ou plutôt la stagnation) de Veneffles par rapport à ce nouveau voisin aux ambitions plus fortes. Sur Amanlis il aurait également été possible de faire mention - ne serait-ce, peut-être, que pour la critiquer - de l'hypothèse de la présence, à l'emplacement du logis noble actuel, d'un château ancien, peut-être construit au XI ${ }^{\mathrm{e}}$ siècle et aujourd'hui détruit ${ }^{11}$ ou encore de la présence d'une probable motte ${ }^{12}$ et de nombreux habitats d'élites à l'ouest du bourg.

Sans doute l'auteur est-il conscient que l'analyse morphologique implique une " certaine profondeur chronologique " qui dépasse le cadre de son étude centrée sur le Moyen Âge (p. 25) et que les " notices de l'atlas morphologique ne correspondent pas à des monographies détaillées" (p. 26). Peut-on le suivre alors lorsqu'il conclut : "Si l'analyse morphologique n'offre qu'une partie des réponses aux questions concernant l'essor et le développement des localités, villages et villes, la morphogenèse nous paraît pour le moment une impasse " (p. 25) ? À mon sens, c'est une autre approche qu'il faut désormais développer, plus archéogéographique qu'historique (au sens classique), c'est-à-dire qui s'affronte réellement à la complexité de l'analyse des formes et en développe tous les raffinements méthodologiques, techniques et épistémologiques. En somme, si cet atlas représente un (gros et ingrat donc respectable) travail, désormais incontournable à tout médiéviste ou moderniste travaillant sur l'histoire de la Haute-Bretagne, on attend que son auteur en étoffe, dans le détail, l'analyse des principaux dossiers.

Magali WatTEAUX (université Rennes 2)

Marchand G., 2014 - Préhistoire Atlantique, fonctionnement et évolution des sociétés du Paléolithique au Néolithique, Arles, Errance, 520 p. (ISBN 978-2-87772-567-5).

Grégor publie ici son mémoire d'Habilitation à Diriger des Recherches sur la période mésolithique sur la façade atlantique. Ouvrage de la maturité, il traite les données accessibles avec une prudence qui l'honore et sans pousser les hypothèses au-delà du raisonnable. S'ajoutent à cela l'emploi d'un vocabulaire riche et précis, parfois incisif et toujours l'emploi d'un humour fin qui lui est propre.

L'auteur entame son ouvrages par un retour sur les archéologues qui ont initié la recherche sur la période et ceux qui l'ont prolongé jusqu'à aujourd'hui. L'auteur, luimême partie prenante de la recherche depuis plus de 25 ans, a traversé les derniers développements de cette recherche en se partageant de façon méritoire et rare au niveau national (toutes périodes confondues) entre archéologie programmée et préventive. Il décrit la diversité des archéologues et leur personnalité scientifique qui vont conditionner la recherche durant des épisodes longs, de l'ordre d'une génération et vont à regret maintenir cette période dans des carcans

10. Poilpré P., ZAC de la Perdriotais, Châteaugiron (35). Étude archivistique, rapport d'étude historique, Inrap, Cesson-Sévigné, 2010, p. 11-14.

11. Châteaux et manoirs du pays de Châteaugiron, Publication de l'Association historique du pays de Châteaugiron, Mémoire n 1, 1993, p. 12.

12. Information Patriarche et toponymie. difficiles à briser, ce qui contribue à la vision toujours (encore) misérabiliste de la période.

Il offre ensuite un panorama des connaissances sur l'évolution naturelle des paysages, faune et flore durant le Tardiglaciaire et le Mésolithique, rendant compte des deux phénomènes majeurs de la période : la remontée de la mer et le remplacement de la faune et de la flore froides par leurs équivalents tempérés. Elle regroupe également les questionnements sur les évolutions culturelles majeures : premier/second Mésolithique et premier Néolithique, en interrogeant sur quelques faits majeurs qui seront discutés plus avant, constituant les éléments les plus avancés mais toujours polémiques concernant la période : céramique ou passage du premier au second Mésolithique sous la poussée des néolithiques orientaux par exemple.

La seconde partie s'ouvre sur la période Tardiglaciaire, qui s'étend de l'Alleröd au début (milieu?) du Préboréal. Ce chapitre regroupe les études des sites fouillés par l'auteur ainsi que ceux de N. Naudinot. Etant donné l'extension de cette période (plus de 5 millénaires), les séries de référence restent encore limitées, même si la dernière décennie a permis d'établir un référentiel typologique et technologique. Les mauvaises conditions de préservation des 